\title{
RESURRECTING GRADUATE CONVERSATION THROUGH AN ONLINE LEARNING COMMUNITY
}

\author{
Brian Thoms, Nathan Garrett, Mariana Soffer, Terry Ryan \\ Social Learning Software Lab, Claremont Graduate University \\ Claremont, California, USA \\ \{Brian.Thoms, Nathan.Garrett, Mariana.Soffer,Terry.Ryan\}@cgu.edu
}

\begin{abstract}
This paper reports on action research that implements online learning community (OLC) software to foster conversation at a specific graduate school. The design of the research is informed by theories of conversation, online learning, and social networking, as well as by popular Web 2.0 technologies. A distinguishing feature of this application is that it is oriented towards and controlled by individuals, rather than being centered on courses. Results indicate that stakeholders-graduate students and faculty_appreciate and find value in the OLC we implemented.
\end{abstract}

\section{KEY WORDS}

Action Research, Conversation, Social Networking, Elgg

\section{INTRODUCTION}

The leaders of our school, a graduate school in the U.S., believe that its continued success depends on the existence of a vibrant intellectual conversation among its stakeholders-students, faculty, staff, and alumni. Unfortunately, recent trends (primarily a decrease in the presence of stakeholders on campus) have led to a reduction in the vitality of this essential conversation. To help foster and revive it, these same leaders have asked our research group to design and implement an information technology (IT)-based solution.

Our guiding philosophy has been to allow people to say that they want to say, to listen to what they want to listen to, to increase their understanding both of themselves and of their fellow community members, and to do it all without having to spend an increased amount of time on campus. To this end, we have implemented software designed to promote free expression of identity and ideas, by and between individuals. Our intent has not been to replace face-to-face interactions, but to supplement them with a persistent virtual component.

After Phase I of our research cycle, we believe that we have achieved a measure of success in improving conversation at our school and also in learning how conversation can be promoted by IT. This paper presents an explanation of the nature of our school's problem, what we did to design and implement a solution, the effect of the solution on our school to date, what is planned for the future and how our work contributes to research.

\section{BACKGROUND}

\subsection{Bringing Conversation Online}

Etched on the perimeter wall of our school there is a phrase, "The center of a college is in great conversation and out of the talk of the college life springs everything else." This observation is widely accepted at our school, especially by our project stakeholders, but it has some problems as a compass for taking action. 
Conversation (to say nothing of great conversation) is difficult to define, even when viewed in terms of a specific population, in our case graduate students, faculty, administrators and alumni. To make progress, we adopted a simple initial working definition of conversation as purposeful (that is to say, related to graduate school activities) peer-to-peer talk. More formally, conversation is a speech exchange system that is structured around turn-taking, a sequential organization of who gets to say what and when [Sacks et al. 1974]. Because speech acts need not be oral or face-to-face, conversation need not be, either. Within this view of conversation as structured speech, not necessarily involving face-to-face talking, it is possible to distinguish a number of purposes for conversation in graduate education. Jenlink and Carr [1996] identify four types of conversation with varying degrees of applicability in a graduate setting:

Conversation as dialectic with focus on logical argument and distilling truth.

Conversation as discussion where many people advocate for their own individual positions.

Conversation as dialogue with focus on constructing meaning through multiple perspectives.

Conversation as design with goals and a focus on creating something new.

Although these notions of conversation are not void at our school, they have been largely confined to within the campus setting. As IT researchers in a largely brick and mortar academic institution, we looked for ways where software has been and can be used to bring conversation into the $21^{\text {st }}$ century. In any acceptable solution, stakeholders would have to be more involved in all conversations, both face-to-face and online.

Most prior research about online conversation focuses on problem solving, decision-making and discussion depth [Sherry 2000]. Although these matters are important, they do not directly address the goals of our project. In a graduate school setting, conversation must extend beyond simple interactions, allowing people to wrestle with complex problems from multiple perspectives. In considering what to do to help our school, we concluded that a more novel approach would be needed to support conversation.

Inspired by outstanding examples of online conversation in a number of very popular online social networking (OSN) applications-including MySpace ${ }^{\mathrm{TM}}$, LinkedIn ${ }^{\mathrm{TM}}$, and Classmates ${ }^{\mathrm{TM}}$-we focused our efforts on the Web 2.0 technologies that they use. These technologies, such as blogs, wikis and peer-to-peer linking, provide the capabilities for users to participate in online conversations, as exemplified by popular OSNs.

OSNs offer an intriguing solution because they employ social ties among friends, and friends-of-friends, to aid in information exchange. OSNs are also intriguing because of their distinction from more traditional course management systems (CMSs). Unlike CMSs, OSNs let individuals own their space, develop their own communities, and control their own participation. In CMSs, control of most aspects of usage rests with course instructors, not students. Furthermore, OSNs make it easy to allow non-student stakeholders to participate, unlike CMSs, which are generally restricted to students enrolled in the particular course.

With these considerations, we decided that some kind of educational OSN application, one that could support stakeholder-defined online learning communities (OLCs), would be our best option for increasing conversation at our school. The largest differentiator between an OSN and OLC is the focus on learning. Although learning can occur in an OSN, the primary objective of an OLC is to foster learning through the community.

\subsection{Online Learning and Online Community}

Studies have shown that interaction and dialogue are essential for productive learning [Cook 2002; Nicol et al. 2003; Sorenson \& Tackle 2002]. Additionally, Internet and multimedia technologies continue to play increasing roles in reshaping the way knowledge is delivered, providing valid alternatives to traditional classroom learning [Garrison 2002; Zhang 2004]. Ultimately, online learning offers just one more approach to learning in addition to new ways of collaborating [Haase 2005].

Wu \& Hiltz [2004] used a questionnaire to examine students' perceptions of their learning after using online discussions. They found that students who were more motivated and enjoyed their experiences also reported higher perceptions of their learning achievements, although they recognized that there may be a difference between perceived and actual learning. Webb et al. [2004] have also noted a positive association between participation in what is termed 'e-learning dialogues' and learning outcomes. 
An OLC must also foster community, often measured in terms of social capital. In a virtual setting, social capital is a common social resource that facilitates information exchange, knowledge sharing, and knowledge construction through continuous interaction, built on trust and maintained through shared understanding [Schwier and McCalla 2003]. Huysman and Wulf [2005] propose that the higher the level of social capital, the more members are stimulated to connect and share knowledge. This sharing aspect challenges an individual to draw upon and provide value for themselves and the community. Social resources often include common identity, familiarity, trust, and a degree of shared language and context among individuals [Lesser and Prusak 1999].

\subsection{Theory}

The primary objective of our software is to promote stakeholder-defined OLCs, which amount to computer-based and learning-oriented communities of practice. A community of practice $(\mathrm{CoP})$ is a group that works together towards common goals, collaborating on common problems, sharing best practices, supporting one another and sharing a common identity [Lave and Wenger 1991]. Critical for the specific knowledge needs of a CoP is that knowledge is self-generating and perpetuating, and transfer is an intrinsic aspect of its functioning [Adams and Freeman 2000]. In creating a sustainable CoP at our school, we draw on theories of learning and community to guide our research.

\subsubsection{Social Constructivism, Social Presence Theory and Activity Theory}

Online learning theories are used to identify ways in which individuals learn. At the graduate level learning is largely self-directed. Social constructivism views each learner as a unique individual with unique needs and backgrounds, both complex and multidimensional [Gredler 1997]. Social constructivism encourages, utilizes, and rewards these characteristics througout the learning process [Hagstrom and Wertsch 1997]. An OLC is a usercentric and user-driven tool offering each user control over his or her own space. As a principal premise of our OLC design, users are the central component of a system that fosters individual representation and particpation in the larger community of practice $(\mathrm{CoP})$ and encouraging users to explore and take ownership of the OLC is critical for its success.

An equally critical component for a successful OLC is the existence of high levels of social capital within the CoP. Social Presence Theory looks at the degree to which an individual's perception of an online community affects his or her participation in that community. Several factors come into play when measuring social presence centered on social context, online communication, and interactivity [Tu 2002]. These components range from group attitudes towards online communication to more personal attitudes on privacy and risk. Research has shown that social presence is one of the most significant factors in improving instructional effectiveness and building a sense of community. Stacey [2002] determined that establishing social presence is an important aspect for effective online interaction and learning and found that a high quality of electronic communication helps to engage students and aids in their learning of the course material.

Activity Theory also applies and can be used as a lens for understanding sociotechnical interactive networks as a function of technology, community development and user interaction between the two. In Activity Theory, activities are goal-directed, where there exists multiple ways to achieve those goals, oftentimes through adaptation [Bødker 1989]. As users begin to adapt to new technologies, they adapt their activities in order to meet the required objectives. In a study on higher education, Issroff and Scanlon [2002] found that Activity Theory forces instructors to consider multiple factors that can impact a specific technologies usage.

\section{ACTION RESEARCH (AR)}

\subsection{Research Design}


The AR approach is highly appropriate for implementing and measuring our software since a sustainable OLC will involve improvements and modifications in order to realign the software with stakeholder objectives. Modifications are derived from the attitudes of course instructors, students, as well as trends in research and popular online networks. This paper focuses on Phase I of the AR cycle, detailing the planning (i.e. analysis and design), implementation and evaluation of our OLC.

Phase I targets a specific population at our school, the transdisciplinary courses (t-courses) taken by all second-year doctoral students from diverse disciplines. These courses are each one semester long and are taught by faculty from all disciplines in our school. Our high-level research question asks how technology can promote conversation. More specific questions focus on the roles learning and community play in conversation at graduate school, and primarily:

1. Can an OLC enable learning among doctoral students?

2. Can an OLC foster community at our school?

3. Will our implementation of an OLC be accepted at our school?

4. Will our OLC implementation be able to sustain an ongoing user population?

\subsection{Elgg Online Learning Landscape and Web 2.0}

Members of an OLC should be able to state what they think, comment on what others have said, collaborate on common statements, and share information in many forms. Web 2.0 technologies, such as blogs, wikis, and peerto-peer file sharing support these activities. And increasingly, individuals are becoming more familiar with these technologies, making their introduction into the classroom more-or-less seamless. As users of advanced learning environments have begun to expect such capabilities, a number of solutions that integrate blogs, wikis and file sharing have become available.

During the planning stage of our AR project we evaluated a variety of proprietary and open source software solutions. We compared technologies based on cost, usability, extensibility, customizability and the range of features each offered. We ultimately decided on the Elgg platform, which labeled itself as an online learning landscape. In addition to the common Web 2.0 utilities such as blogging, file sharing and peer networking, Elgg offers individuals and communities the ability to restrict access to data on a number of levels (individual, community, logged in users or custom levels). Elgg also allows for individual customization, allowing users to create more personalized pages.

Beyond these functional requirements, extensibility was a critical factor in our choice of platform. The Elgg open source software provided us with the freedom to development new components and to customize the software to meet the needs of our school, as well as our research objectives. As one example, the requirement to support individual portfolio building and group writing led to the addition of the wiki add-in. Although Elgg did not provide wiki functionality, it was extensible enough that we were able to add what was needed.

\subsection{OLC Implementation}

During the analysis and design phase of our project, we met regularly with project stakeholders and course instructors for training and general discussion on how an OLC could augment classroom experiences. Although course instructors were not mandated to use our software, we worked closely to showcase the benefits of using a combination of traditional classroom techniques with a virtual component to further extend the classroom conversation. More specifically, we focused on how course syllabi could be aligned with our software. For example, if a course required weekly assignments based on selected course readings, or guest lectures, we recommended the community blog as a viable option for individuals to express themselves. For more collaborative projects, we recommended that students use the wiki. In the end, it was left up to the instructor to decide how best to use the technologies and our role was to help facilitate instructors in achieving their specific objectives. The result was $100 \%$ adoption by t-course instructors.

Our group sessions also provided feedback on what additional features should be incorporated and helped to identify system bugs as well. After three months of development, our tool was a hybrid of in-house and Elgg- 
bundled software, hosted on a local web server and customized for our specific school. Table 1 provides a list of Web 2.0 technologies that comprised our tool. Each technology, with the exception of the chat feature, used sparingly, played an integral part in learning and forming community.

Table 1. Features of the extended-Elgg OLC.

\begin{tabular}{|l|l|}
\hline Bundled with Elgg & Add-in \\
\hline Profile building & Collaborative writing (wiki) \\
\hline Blogging & Enhanced commenting for blogging \\
\hline File repository & Enhanced commenting on wiki \\
\hline Networking capabilities & Chat Room (added post-release) \\
\hline
\end{tabular}

\section{SPECIFYING LEARNING}

During the first semester of implementation we focused on providing a stable OLC. During this time we also measured acceptance of the OLC, how it aided in learning, and how it helped in building community. Although we released the tool to a wider population of courses, we focused primarily on the t-courses for analysis, which by their nature place a high emphasis on collaboration and discourse.

\subsection{Pretest Analysis}

We conducted an initial pretest to capture general information about our user population prior to their using the tool. The pretest, a closed ended questionnaire, yielded 51 usable responses $(62 \%)$ from our initial pool of $82 \mathrm{t}$ course participants.

\subsubsection{Predicting Online Success, Technology Familiarity and Social Tendencies}

The first measurement looked to predict the success of our OLC through a general online compatibility scale. The instrument was designed primarily to assess the willingness of an individual to use online methods of learning and communicating. Developed by Smith et al. [2003] and consisting of 22 items, each scored on a 5-point scale, the average rating was 2.16 indicating that, on average, users would have minimal resistance to learning and communicating through online methods.

Table 2. Technology familiarity.

In addition to online readiness, we wanted to know how familiar individuals were with many of the technologies incorporated in our software. With the exception of RSS and wiki technologies, the majority of users were familiar or somewhat familiar with our software's technologies. Table 2 displays these results.

As one last pretest measure, we included a general sociability index based on an introversion/extroversion assessment technique developed by an in-house expert.

\begin{tabular}{|l|l|l|l|}
\hline \multirow{2}{*}{ Technology } & \multicolumn{3}{|c|}{ Familiarity (in \%) } \\
\cline { 2 - 4 } & $\begin{array}{c}\text { Very } \\
\text { Familiar }\end{array}$ & $\begin{array}{c}\text { Somewhat } \\
\text { Familiar }\end{array}$ & $\begin{array}{c}\text { Not } \\
\text { Familiar }\end{array}$ \\
\hline Blogging & $33 \%$ & $53 \%$ & $14 \%$ \\
\hline E-Profiles & $20 \%$ & $43 \%$ & $37 \%$ \\
\hline File Sharing & $31 \%$ & $41 \%$ & $28 \%$ \\
\hline RSS & $12 \%$ & $26 \%$ & $63 \%$ \\
\hline Social Networks & $39.5 \%$ & $29.5 \%$ & $31 \%$ \\
\hline Wiki & $23.5 \%$ & $27.5 \%$ & $49 \%$ \\
\hline
\end{tabular}

The questionnaire measures an individual's sociability based on responses to 17 questions, each with two choices (one indicating an extroverted action, the other an introverted response). The average rating of 1.54 indicated a balance of introverts and extroverts.

\subsection{Usage Data}


Table 3. Site usage: t-course vs. all.

To get a general indication of the impact our software was having, we used indirect data collection based on database updates. Table 3 shows side-by-side comparison of technology usage across our school. In this research we focus on t-course usage, comprising roughly two-thirds of the site participants. These users accounted for the majority of file uploads (60\%), wiki edits $(64 \%)$ and wiki comments $(62 \%)$. However, the same two-thirds accounted for less total blog additions

\begin{tabular}{|l|c|c|c|}
\hline \multicolumn{1}{|c|}{ Category } & T-Courses & All & As \% \\
\hline Users & 82 & 130 & $63 \%$ \\
\hline Communities & 16 & 29 & $55 \%$ \\
\hline Blogs & 486 & 1154 & $42 \%$ \\
\hline Blog Comments & 422 & 986 & $43 \%$ \\
\hline Wiki Pages (All) & 907 & 1427 & $64 \%$ \\
\hline Wiki Pages (Unique) & 127 & 356 & $36 \%$ \\
\hline Wiki Comments & 23 & 37 & $62 \%$ \\
\hline File Uploads & 115 & 193 & $60 \%$ \\
\hline
\end{tabular}
$(42 \%)$ and blog comments (43\%) with less unique wiki page contributions as well $(36 \%)$.

\subsection{Posttest Analysis}

The posttest questionnaire focused on assessing online community, social learning and specific technologies, in addition to overall OLC satisfaction. Closed- and open-ended questions, distributed to the same population of tcourse participants (81 individuals), resulted in 50 (61\%) usable responses, 35 of which also responded with qualitative data.

\subsubsection{Overall User Experience}

Table 4. Assessing overall experience $(n=50)$.

A 5-point scale measured the impact of our tool. Overall, the OLC was positively received and the majority of participants agreed that the tool was an important edition to their course. Table 4 provides a snapshot of our data, where only $12 \%$ of respondents indicated that they were dissatisfied with the tool. $58 \%$ were satisfied or strongly satisfied with the software, while $30 \%$ remained neutral. Although $40 \%$ of the

\begin{tabular}{|l|c|c|c|c|c|}
\hline 1=Strongly Agree, 2=Agree, 3=Neutral, 4=Disagree, 5=Strongly Disagree \\
\hline \multicolumn{1}{|c|}{ Survey Item } & $\mathbf{1}$ & $\mathbf{2}$ & $\mathbf{3}$ & $\mathbf{4}$ & $\mathbf{5}$ \\
\hline Overall software satisfaction & $22 \%$ & $36 \%$ & $30 \%$ & $12 \%$ & $0 \%$ \\
\hline Achieved class objectives & $19 \%$ & $41 \%$ & $30 \%$ & $10 \%$ & $0 \%$ \\
\hline More interaction (professor) & $14 \%$ & $24 \%$ & $22 \%$ & $16 \%$ & $24 \%$ \\
\hline More interaction (peers) & $25 \%$ & $30 \%$ & $27 \%$ & $13 \%$ & $5 \%$ \\
\hline Strengthened relationships & $22 \%$ & $25 \%$ & $30 \%$ & $14 \%$ & $8 \%$ \\
\hline Should have an OLC & $24 \%$ & $43 \%$ & $30 \%$ & $3 \%$ & $0 \%$ \\
\hline Prefer over traditional CMS & $24 \%$ & $30 \%$ & $32 \%$ & $12 \%$ & $0 \%$ \\
\hline Consider use outside the course & $8 \%$ & $39 \%$ & $31 \%$ & $14 \%$ & $8 \%$ \\
\hline Importance of a .EDU extension & $31 \%$ & $40 \%$ & $22 \%$ & $3 \%$ & $3 \%$ \\
\hline
\end{tabular}
respondents felt they had less interaction with their instructor, 55\% reported more interaction with peers than in other classes. An important aspect of the t-course is in building peer-to-peer relationships and $47 \%$ reported a strengthening in peer-relationships compared with other courses. It was largely encouraging to find that only $3 \%$ of respondents disagreed with the notion of an OLC at our school, and 67\% agreed or strongly agreed.

Lastly, over $50 \%$ felt that our software was preferred over traditional CMSs and that an academic OLC is an important addition to the graduate experience. Along these lines, the general consensus was that our software should be hosted under an EDU domain (71\%), as opposed to our choice for an ORG extension, implying that an institutionally backed tool would be more appropriate.

\subsubsection{Community Assessment}

We also measured how effective our OLC was in building community and fostering social interaction. The responses we received were encouraging and largely positive. Based on a 6-point scale, $82 \%$ of respondents viewed the OLC as an excellent medium for social interaction. Additionally, we found that the majority of respondents were comfortable introducing themselves $(88 \%)$, conversing $(84 \%)$ and interacting with others (92\%) through the OLC. The OLC also helped individuals form distinct impressions of one another (86\%). Additionally, respondents felt that the community helped to improve their learning (78\%). Table 5 provides a complete set of responses. 
Table 5. Assessing community and effectiveness $(n=50)$.

\begin{tabular}{|c|c|c|c|c|c|c|c|}
\hline Survey Item & 1 & 2 & 3 & 4 & 5 & 6 & NA \\
\hline Was an excellent medium for social interaction & $8 \%$ & $36 \%$ & $38 \%$ & $4 \%$ & $10 \%$ & $2 \%$ & $2 \%$ \\
\hline Felt comfortable conversing through this medium & $14 \%$ & $50 \%$ & $20 \%$ & $12 \%$ & $2 \%$ & $2 \%$ & $2 \%$ \\
\hline Felt comfortable introducing myself in this course & $16 \%$ & $42 \%$ & $30 \%$ & $10 \%$ & $0 \%$ & $0 \%$ & $2 \%$ \\
\hline The medium helped me feel like part of the community & $12 \%$ & $22 \%$ & $50 \%$ & $6 \%$ & $6 \%$ & $12 \%$ & $0 \%$ \\
\hline Was comfortable participating in course discussions & $26 \%$ & $38 \%$ & $24 \%$ & $4 \%$ & $8 \%$ & $0 \%$ & $0 \%$ \\
\hline Was comfortable interacting with other participants in the course & $32 \%$ & $34 \%$ & $26 \%$ & $4 \%$ & $4 \%$ & $0 \%$ & $0 \%$ \\
\hline My point of view was acknowledged by others in the course & $20 \%$ & $38 \%$ & $24 \%$ & $12 \%$ & $4 \%$ & $0 \%$ & $2 \%$ \\
\hline Was able to form different impressions of course participants & $20 \%$ & $40 \%$ & $26 \%$ & $8 \%$ & $2 \%$ & $0 \%$ & $4 \%$ \\
\hline A strong sense of community existed in this course & $24 \%$ & $20 \%$ & $28 \%$ & $10 \%$ & $12 \%$ & $4 \%$ & $2 \%$ \\
\hline Course community improved my learning & $20 \%$ & $28 \%$ & $30 \%$ & $8 \%$ & $6 \%$ & $8 \%$ & $0 \%$ \\
\hline
\end{tabular}

\subsubsection{Technology Assessment (Quantitative and Qualitative Data)}

Quantitative and qualitative data measured how specific technologies enhanced learning, social interaction and community. Using the same scale as in Section 4.3.2, users ranked each technology on the criteria of how well each fostered these three areas. Overall, users agreed (average rating of 2 and 3) that the ability to blog and comment on other blogs helped across all areas. Similarly, wiki writing and sub-communities also helped to improve learning, social interaction and community building (average rating of 2).

Qualitative questions allowed individuals to elaborate on various aspects of the system and will, in turn, help us improve our software going forwards. When asked what features worked best, individuals responded closely stating that the ability to view peer work and work collaboratively were strong points of the system. One response stated, "My favorite part was that I could see other people's work, which allowed me to learn from them." Another response stated, "I think it's beneficial to share knowledge \& opinions w/ classmates and the [software] helped support these sharing activities." Responses on site recommendations and improvements ranged broadly. Some recommended more customizable profile pages, similar to popular social networking websites, while others requested interactive online tutorials. Lastly, a theme developed on fixing and improving existing site navigation and functionality for greater ease of use.

\section{CONCLUSION AND FUTURE WORK}

Over the past eight months, our project team has added a virtual component to the scholarly conversation at our school. 260 graduate students and faculty currently participate with new members joining daily. Our tool has achieved a measure of success in providing an asynchronous online component for students and faculty to collaborate and share knowledge in courses and on external projects. Guided by high-level theories of learning and social networking, direct and indirect data indicates a user-driven OLC to be beneficial to learning at the graduate level. Should our results hold across subsequent populations, the OLC approach may provide a solution to increased scholarship at our school as well as a valid alternative to more traditional CMS solutions. Additionally, the initial success has provided a proof-of-concept for a more publicized rollout and integration with our school's infrastructure for the 2007-2008 academic year.

As we enter Phase II of our AR project we look to build on and improve numerous aspects of the system. Additionally, although the initial acceptance of our tool is a critical issue in determining whether it is successful, its continued use is at least as important [Bhattacherjee 2001]. Therefore, we will continue to monitor how our tool is adopted and used across our school, looking for ways to extend its use, including to alumni who are also interested in continuing the scholarly conversation. 


\section{REFERENCES}

Adams E.C. and Freeman C., 2000. Communities of practice: bridging technology and knowledge assessment. Journal of Knowledge Management. Vol. 4 No. 1, pp. 38-44.

Bødker, S., 1989. A human activity approach to user interfaces. Human-Computer Interaction, Vol. 4, pp. $171-195$.

Bhattacherjee, A., 2001, Understanding information systems continuance: An expectation-confirmation model. MIS Quarterly, Vol. 25, No. 3, pp. 351-370.

Cook, J., 2002. The Role of Dialogue in Computer-Based Learning and Observing Learning: An Evolutionary Approach to Theory. Journal of Interactive Media in Education.

Daniel, B. et al, 2003. Social Capital in Virtual Learning Communities and Distributed Communities of Practice. Canadian Journal of Learning and Technology, Vol. 29 No. 3.

Garrison, D. R., 2002. Cognitive Presence for Effective Asynchronous Online Learning: The Role of Reflective Inquiry, Selfdirection and Metacognition Fourth Annual Sloan ALN Workshop.

Hagstrom, F. and Wertsch, JV, 2004. The Social Construction of identity: The Clinical Challenge. Topics in Language Disorders. Vol. 24, No. 3 pp. 162-173.

Huysman and Wulf, 2005. Information Technology in Building and Sustaining the Relational Base of Communities, The Information Society, Vol. 21, pp. $81-89$.

Issroff, K., and Scanlon, E., 2001. Case studies revisited- what can Activity Theory offer? Proceedings of First Euro-CSCL Conference, Maastricht.

Jenlink, P., and Carr, A.A, 1996. Conversation as a medium for change in education. Educational Technology, pp. 31-38.

Lave, J and Wenger, E., 1991. Situated Learning: Legitimate Peripheral Participation. Cambridge University Press.

Lesser, E., and Prusak, L., 2000. Communities of practice, social capital and organizational knowledge. Knowledge and communities pp. 123-131.

Nicol, David et al, 2003. The Social Dimensions of Online Learning. Innovations in Education \& Teaching International, Vol. 40, Issue 3, pp 270-280.

Haase, Anabel, Trends in Online Learning Communities, ACM SIGGRROUP Bulletin, Special Issue On Online Learning Communities Vol. 25 pp. 2-6.

Sacks, H., Schegloff, E. A., and Jefferson, G., 1974. A Simplest Systematics for the Organization of Turn-Taking for Conversation. Language, Vol. 50, No. 4, pp. 696-735.

Sherry, L, 2000. Good online conversation: Building on research to inform practice. Journal of Interactive Learning Research, Vol. 11, Issue 1, p85.

Smith P.J. et al, 2003. Towards Identifying Factors Underlying Readiness for Online Learning: An Exploratory Study. Distance Education, Vol. 24, No. 1, pp. 57-67.

Stacey, E., 2002. Social Presence Online: Networking Learners at a Distance. Education and Information Technologies, Kluwer Academic Publishers, Vol. 7, No. 4, pp. 287-294.

$\mathrm{Tu}, \mathrm{CH}$, The measurement of social presence in an online learning environment. International Journal of eLearning, corporate, Government, Healthcare, \& Higher Education, AACE, Vol. 2 No. 1 pp. $34-45$.

Webb, Eileen, et al, 2004. Using e-learning dialogues in higher education. Innovations in Education and Teaching International, Vol. 41, No. 1.

Wu, D. and Hiltz, S., 2004. Predicting Learning from Asynchronous Online Discussions, Journal of Asynchronous Learning Networks, pp. 139-152.

Zhang, D, 2004. Can e-learning replace classroom learning? Communications of the ACM, Vol. 47 p75. 